\title{
Trivium
}

Revue franco-allemande de sciences humaines et sociales - Deutsch-französische Zeitschrift für Geistesund Sozialwissenschaften

$14 \mid 2013$

Le Saint-Empire à l'époque moderne

\section{Chevalerie immédiate d'Empire et tribunal de la Chambre impériale}

\section{Heinz Duchhardt}

Traducteur : Christophe Duhamelle

\section{(2) OpenEdition}

Journals

Édition électronique

URL : http://journals.openedition.org/trivium/4609

DOI : $10.4000 /$ trivium.4609

ISSN : 1963-1820

Éditeur

Les éditions de la Maison des sciences de l'Homme

Référence électronique

Heinz Duchhardt, «Chevalerie immédiate d'Empire et tribunal de la Chambre impériale », Trivium [En ligne], 14 | 2013, mis en ligne le 16 septembre 2013, consulté le 08 septembre 2020. URL : http:// journals.openedition.org/trivium/4609; DOI : https://doi.org/10.4000/trivium.4609

Ce document a été généré automatiquement le 8 septembre 2020

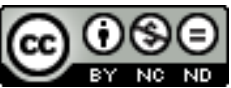

Les contenus des la revue Trivium sont mis à disposition selon les termes de la Licence Creative Commons Attribution - Pas d'Utilisation Commerciale - Pas de Modification 4.0 International. 


\title{
Chevalerie immédiate d'Empire et tribunal de la Chambre impériale
}

\author{
Heinz Duchhardt \\ Traduction : Christophe Duhamelle
}

\section{NOTE DE L'ÉDITEUR}

Nous remercions M. Heinz Duchhardt ainsi que la maison d'édition Duncker \& Humblot de nous avoir accordé l'autorisation de traduire ce texte pour le présent numéro.

Wir danken Herrn Heinz Duchhardt und dem Verlag Duncker \& Humblot die freundliche Genehmigung, diesen Artikel in französischer Übersetzung zu publizieren.

\section{NOTE DE L'AUTEUR}

Cet article constitue la version augmentée d'une conférence présentée le 21 janvier 1977 à l'université d'Augsbourg. Il repose entre autres sur des archives manuscrites du Staatsarchiv de Darmstadt (StADa), du Landeshauptarchiv de Coblence (LHAKo) et du Haus-, Hof- und Staatsarchiv de Vienne (HHStAW). Il va de soi que cette première esquisse ne saurait épuiser en détail tout le spectre des relations que la chevalerie immédiate d'Empire entretenait avec le tribunal de la Chambre impérial (abrégé en TCI).

En septembre 1522, le chevalier Franz von Sickingen campait avec son armée devant la ville de Trèves. Aux états et au gouvernement de l'Empire qui ne cessaient de l'adjurer de choisir la voie judiciaire pour régler le conflit qui l'opposait à l'archevêque-électeur, il répondit par la dérision, se faisant l'écho des griefs accumulés contre les jeunes instances de la justice impériale : "Ils ont à Nuremberg un tribunal de la Chambre, il est vrai, mais lui ${ }_{58}^{F_{B}}$ Sickingen ${ }_{50}$ a un tribunal itinérant qui se distingue par ses douilles et ses mortiers et il entend y faire son procès, de simplici ad plenum ${ }^{1}$. Quelques mois après le déclenchement de la Révolution française, le canton de chevalerie du Rhin-Moyen fit connaître son souhait d'obtenir le droit de présenter au titre de l'organisation 
d'ensemble de la chevalerie immédiate un certain nombre d'«assesseurs » (juges) du tribunal de la Chambre impériale [TCI] et d'en assurer le financement ${ }^{2}$.

2 Entre ces deux documents, il n'y a pas seulement plus de deux siècles et demi d'écart; on change véritablement d'univers. Comment se fait-il que les libres chevaliers et leur corporation archaïque en soient venus à modifier si radicalement leur rapport à l'institution incarnant la justice d'Empire ? La question est bien celle de la nature et de l'évolution des relations entre la libre et immédiate chevalerie d'Empire et le tribunal de la Chambre impériale au cours de l'époque moderne - la chevalerie qui depuis le troisième quart du XVIe siècle, pour se prémunir contre les appétits des princes territoriaux, voyait dans l'empereur Habsbourg le protecteur et le garant de son existence ainsi que la figure de référence de sa cohésion naissante, à tel point qu'elle se définit elle-même en 1578 comme le « cœur et les membres de l'aigle noir déployé » ${ }^{3}$ et qu'elle ne cessa de se faire confirmer cette relation spéciale avec le monarque dans toutes les capitulations électorales et les autres privilèges ${ }^{4}$. Le TCI quant à lui ne put jamais faire oublier ses origines ancrées dans le système des princes d'Empire et dès la fin du XVIe siècle, parallèlement à l'évolution qui en faisait une instance confessionnellement paritaire, s'érigea en pôle opposé à la cour de Vienne, avec des conséquences de plus en plus néfastes.

3 Le chemin qui permit à la libre noblesse chevaleresque de se doter d'une organisation autonome fut semé d'embûches ${ }^{5}$. Les premières tentatives, dans le sillage de ses efforts réussis pour échapper à l'impôt du denier commun (Gemeiner Pfennig), en 1495, furent un échec. C'est à partir des années 1530 et surtout de la Diète de Spire en 1542 que la chevalerie commença à créer une véritable organisation qui parvint dans les années 1560-1570 à faire inscrire définitivement dans le droit impérial son exemption du système des états d'Empire et de la participation aux Cercles d'Empire*, ainsi que sa soumission immédiate - c'est-à-dire sans intermédiaire féodal - au seul empereur ${ }^{6}$. Cependant, depuis 1495 la chevalerie accueillit les nouvelles institutions (le TCI et toutes celles qui furent créées en ces années de réforme de l'Empire, les Cercles par exemple) avec une grande réserve et même une méfiance marquée. La libre chevalerie avait le sentiment (non sans de bonnes raisons subjectives et objectives) d'être la grande perdante de cette réforme et, préférant bouder dans son coin, elle n'a cessé des décennies durant d'émettre des protestations contre les acquis et les institutions de $1495^{7}$. Elle présenta par exemple une longue liste de griefs à la Diète de Nuremberg en 1523 : l'idée que le TCI était un instrument des princes et offrait à la chevalerie tout sauf une sécurité juridique en était un élément central ${ }^{8}$. On connaît bien d'autres voix qui ont porté les accusations les plus sévères contre le nouveau tribunal central ${ }^{9}$; sa simple existence, et sa vocation à défendre la paix publique, ne pouvaient apparaître aux yeux des chevaliers que comme une attaque frontale contre leur tradition de la faide - autrement dit, comme une tentative d'éliminer le droit traditionnel de la noblesse à régler elle-même ses litiges juridiques. On ne peut se contenter, comme l'a fait une historiographie déjà ancienne $\mathrm{e}^{10}$, de considérer le combat des libres chevaliers contre le Gemeiner Pfennig de 1495 comme la simple expression d'une contradiction fondamentale entre l'imposition et le code d'honneur ou l'identité sociale des chevaliers ${ }^{11}$. Il faut plutôt le voir comme le refus de contribuer à financer l'existence d'un tribunal détesté. Les efforts désespérés des chevaliers au cours des deux premières décennies du XVIe siècle, pour institutionnaliser une procédure de règlement arbitral des conflits qui les opposaient aux princes, sont la preuve supplémentaire de leur 
opposition viscérale au nouveau tribunal ${ }^{12}$, de même que leur vaine tentative, en 1521, pour faire figurer l'interdiction de tout recours au TCI dans l'accord final sur l'ordonnance réglementant le droit d'austrègue ${ }^{13}$.

4 Les remarques dépréciatives dirigées explicitement contre les docteurs en droit issus de la bourgeoisie peuplant le tribunal indiquent toutefois que le malaise des chevaliers allait plus loin que le simple mécontentement face à l'existence d'une instance qui bouleversait leurs habitudes juridiques et ne concernait pas seulement leur nonparticipation à la création et à l'entretien de la Chambre. Ces remarques reflètent en outre la crise plus générale d'identité dans laquelle la noblesse était entrée au cours des mutations structurelles du XVe siècle. Sa prééminence sociale était menacée sur deux fronts : celui de l'émergence des pouvoirs princiers, et celui de l'essor de la bourgeoisie. Il s'agissait de deux dangers jumeaux : la bourgeoisie opposait partout une concurrence acharnée à la noblesse à mesure que se formait l'État territorial de la première modernité ${ }^{14}$. Profitant de sa formation juridique qui la qualifiait pour les tâches nouvelles et variées de l'administration, elle rejetait de plus en plus la noblesse dans l'ombre - dans la mesure où les nobles n'étaient pas disposés à acquérir les mêmes qualifications. C'est là que résidait le défi numéro un de la chevalerie dans la première moitié du siècle de la Réforme: se forger par les études et la formation de quoi remplacer les anciennes prérogatives fondées sur la tradition. C'est le second fait important qui permet d'expliquer pourquoi pendant des décennies les relations entre le TCI et la chevalerie n'ont pas pu s'épanouir ou, pour le dire autrement, sont demeurées à un stade embryonnaire. Car au-delà de l'opposition fondamentale des chevaliers à l'encontre d'un nouveau tribunal imposé et entretenu par les états d'Empire, on aurait pu attendre d'eux qu'ils affichent au moins une teinture de formation juridique, afin de pouvoir occuper les postes de juges qui leur avaient été réservés en $1495^{15}$; mais, à quelques exceptions près, ils en étaient totalement dépourvus. On trouve dans la chronique des Zimmern, à propos du milieu nobiliaire souabe, une remarque qui peut certainement être étendue à l'ensemble de la libre chevalerie: il y régnait "haine et mépris envers les études ${ }^{16}$. L'attitude de la chevalerie d'Empire envers le TCI a été déterminée pendant tout le XVIe siècle par ces deux principes : négation et rejet d'une institution dominée par les états d'Empire d'un côté ; absence de goût et de compétence pour s'y investir sans crainte de déchoir de l'autre. La chronique des Zimmern montre aussi à quel point il était alors malaisé de trouver un noble, et plus particulièrement un chevalier, qui acceptât d'occuper un poste d'assesseur au TCI. Selon ce texte, il fallut l'«insistance répétée » des deux envoyés de l'empereur, manifestement réduits au désespoir, pour que Wilhelm Werner von Zimmern consente finalement à rejoindre pour quelque temps le tribunal de Spire $^{17}$. Il suffit d'ailleurs de signaler que la disposition qui, dans les anciennes ordonnances sur le TCI de 1495, 1507 et 1521, réservait la moitié des postes d'assesseurs à des docteurs en droit et l'autre moitié à des nobles, a dû être abrogée au plus tard en 1555-1557 - en raison, justement, du nombre trop limité de nobles intéressés par la position et suffisamment qualifiés.

5 On peut donc compter sur les doigts des deux mains les chevaliers d'Empire qui occupèrent le poste de juge au tribunal de la Chambre au cours du XVIe siècle - ils ne jouèrent un rôle notable que pendant la toute première phase de consolidation du TCI. Prenons par exemple la décennie 1530-1540 : en 1530, on compte seulement trois nobles parmi les 16 juges: Glauburg ${ }^{18}$, Hirnheim et Dechwitz - dont un seul fait partie de la libre chevalerie. De 1531 à 1540, 45 mouvements de personnel affectèrent les 
assesseurs ; 37 des nouveaux arrivants étaient des bourgeois diplômés, six des nobles médiats dont trois seulement avait obtenu leur doctorat, deux enfin, Philipp von Venningen et le docteur von Riepur, appartenaient à la chevalerie immédiate ${ }^{19}$.

6 Un autre domaine permet de constater l'opposition radicale des chevaliers envers un tribunal fondé sur une initiative qui n'était pas la leur et selon eux créé pour contrer leurs intérêts: il s'agit de la mise en place, par chacun des cantons formant l'organisation corporative naissante de la chevalerie immédiate d'Empire, d'instances judiciaires particulières, ainsi que de leur souci de court-circuiter tout appel au TCI ${ }^{20}$. En tant que membres immédiats de l'Empire, les chevaliers disposaient pourtant du droit personnel de se pourvoir directement devant les tribunaux suprêmes de l'Empire - TCI et Conseil impérial aulique (Reichshofrat) - et également de cette possibilité de médiation amiable par les pairs que l'on appelait le droit d'austrègue et qui leur fut confirmée une fois de plus par l'ordonnance sur la Chambre impériale de 1555. Au XVIe siècle, les chevaliers renoncèrent, ou peu s'en faut, à utiliser le TCI comme tribunal de première instance et ils mirent tout en œuvre pour bloquer sa saisine en appel ${ }^{21}$. Ils confièrent la juridiction sur leurs querelles internes aux directions des cantons, promues ainsi au rang de première instance, ou plus précisément de tribunal d'austrègue permanent ${ }^{22}$. Dans les années 1590 , on en était encore à essayer de mettre sur pied une seconde instance constituée par les directoires des cercles ${ }^{23}$ et censée, en formant le dernier échelon judiciaire possible, rendre totalement superflu le rôle de cour d'appel du TCI. À une date aussi tardive que les années 1650, les ordonnances chevaleresques des cercles de Franconie et de Souabe tentaient toujours d'empêcher le pourvoi devant le TCI en première instance et de n'autoriser sa saisine en appel que pour les litiges correspondant à une somme si considérable que les chevaliers ne pouvaient en fait jamais y avoir recours ${ }^{24}$.

7 À la même époque, vers le milieu du XVIIe siècle, les chevaliers avaient cependant commencé partout à faire machine arrière : aucun de leurs tribunaux d'austrègue ne fonctionnait correctement ${ }^{25}$ et ils ne parvinrent en outre jamais à convaincre les autres membres de l'Empire - ceux qui ne faisaient pas partie de la chevalerie immédiate - de se soumettre à cette procédure arbitrale. Ne serait-ce que pour cette raison, les chevaliers étaient bien obligés de reconsidérer leur aversion viscérale envers le tribunal d'Empire siégeant alors à Spire. Vers 1650 au plus tard, les chevaliers immédiats se virent contraints de se tourner à nouveau vers les institutions impériales en matière juridictionnelle. Le TCI de Spire - établi plus tard à Wetzlar - devint par la suite le lieu où les chevaliers portaient en priorité leurs procès (fort nombreux au demeurant), même en première instance ${ }^{26}$. Il est toutefois nécessaire d'établir des différences régionales et de ne pas négliger la fonction importante dévolue à l'autre tribunal d'Empire, le Conseil impérial aulique ${ }^{27}$. C'est en tout cas en raison de cette évolution que depuis le début du XVIIe siècle la chevalerie immédiate s'était résolue à entretenir auprès du tribunal de la Chambre impériale un procureur chargé de suivre les procès concernant les chevaliers ; la nécessité ne s'en était tout simplement pas fait sentir avant cette date.

Deux initiatives impériales furent à l'origine de cette mutation. En 1566, l'empereur Maximilien II fit savoir à la chevalerie que le TCI serait le conservateur, le rempart et le protecteur de ses libertés ${ }^{28}$. D'autre part, l'habitude s'était établie depuis la fin du XVIe siècle que les empereurs de la Maison d'Autriche fassent obligation au TCI de respecter les privilèges de la chevalerie et l'enjoignent de prendre un soin tout 
particulier des libertés et immunités chevaleresque ${ }^{29}$. Des prescriptions impériales de ce type furent par exemple signifiées au tribunal par Rodolphe II en 1591 et 1605, par Ferdinand II en 1632 et par Ferdinand III en $1654^{30}$. Cette évolution fut couronnée à la fin du XVIIe siècle par le traitement de faveur accordé par l'empereur à la chevalerie d'Empire en matière de procédure (pour les procès simplifiés et la signification des mandats), traitement tout à fait inhabituel qui fit l'objet de violentes attaques de la part des états d'Empire ${ }^{31}$. Autre indice que le vent avait tourné : depuis 1599 la chevalerie irritée par la pratique de l'austrègue entre les princes, qui lui était fortement défavorable, ainsi que par les prétentions que les tribunaux territoriaux élevaient contre elle ${ }^{32}$ - s'était mise à échanger avec les empereurs une abondante correspondance où elle émettait des propositions constructives sur l'amélioration et la réforme des procédures juridiques du tribunal de la $\mathrm{Chambre}^{33}$, donnant ainsi de multiples gages de l'intérêt nouveau qu'elle accordait à l'instance de Spire. Cela est d'autant plus remarquable qu'à cette époque le rôle du TCI était au plus bas - il est vrai que c'est aussi l'époque où la chevalerie avait enfin pris pleinement conscience qu'elle serait toujours perdante si elle n'avait (ou choisissait de n'avoir) aucune instance judiciaire devant laquelle porter ses conflits. D'une manière plus générale, la crise de la constitution de l'Empire, qui culmina dans l'échec de la Diète de 1608, et plus particulièrement dans la violation des droits fondamentaux que constitua l'affaire de Donauwörth en 1607, où l'empereur perdit une bonne partie de son aura de protecteur de la légalité impériale, ont pu constituer pour les chevaliers de bonnes raisons d'aller chercher refuge auprès d'une autre institution consacrée à la défense du droit ${ }^{34}$.

C'est à peu près au même moment - à partir de la guerre de Trente Ans et la paix de Westphalie - que le TCI se mit à présenter aux yeux des chevaliers un attrait supplémentaire et tout à fait nouveau : celui d'un lieu où ils pouvaient faire carrière. Quelles sont les causes de cet investissement professionnel inédit? Deux facteurs semblent dominer. Le premier réside dans les difficultés économiques que les chevaliers partageaient avec l'ensemble de la noblesse et qui, plus menaçantes encore depuis la fin du XVIe siècle, accroissaient pour les fils puînés la nécessité de trouver des situations qui puissent les faire vivre. Le second est d'ordre confessionnel : depuis la guerre de Cologne en 1583 et la querelle du chapitre cathédral de Strasbourg, les protestants avaient dû abandonner l'idée de réviser le réservat ecclésiastique de 1555. Quant aux catholiques, il leur avait fallu (au plus tard au moment de la paix de Westphalie) faire leur deuil de leurs espoirs de voir annuler les sécularisations de biens ecclésiastiques opérées par les protestants. Chaque camp confessionnel voyait ainsi s'échapper de confortables prébendes et en était réduit à chercher des solutions alternatives. Ajoutons l'impression qu'avaient les chevaliers d'être rejetés dans l'ombre par les États territoriaux dont la vigueur ne cessait de croître, et de se retrouver le dos au mur, ayant ainsi besoin que leurs intérêts politiques soient efficacement représentés auprès des institutions d'Empire - ce sentiment a sans doute lui aussi joué un rôle dans la propension nouvelle des chevaliers d'Empire à faire carrière au sein du TCI. Ils cherchaient bien sûr à y occuper d'abord les postes les plus prestigieux, à commencer par celui de président. Leurs efforts, à partir du milieu du XVIIe siècle, pour obtenir au tribunal des positions directement conférées par l'empereur, socialement valorisantes et somme toute compatibles avec l'ethos nobiliaire, furent couronnés de succès - du moins pour certains lignages comme les Dalberg, les Ingelheim, les Groschlag, les Waldbott-Bassenheim, les Thüngen et les Seckendorff. Rien ne montre davantage le contraste avec la totale inappétence des chevaliers envers ces carrières dans la période 
précédente que les innombrables et véhémentes protestations - et leur sillage de libelles ${ }^{35}$ - que les comtes d'Empire émirent contre cette intrusion d'une concurrence aristocratique dans un domaine qui jusque-là avait pu passer pour leur pré carré3 ${ }^{36}$. En effet, cela faisait des décennies que la pratique constitutionnelle, sinon le droit, faisait de la qualité d'état d'Empire une condition absolue pour l'obtention de ces postes. Mais les chevaliers ne cessaient désormais de donner des coups de canif à ce principe. On voit ainsi dans les décennies qui suivent 1650 faire irruption parmi les assesseurs du TCI des chevaliers issus des familles Faust von Stromberg, Haxthausen, Ritter von Groenstein, Buwinghausen, Riedesel, Walderdorff, Gudenus, Künßberg et Gemmingen ${ }^{37}$. $\mathrm{Au}$ sein de la chevalerie franconienne, on débattit ouvertement en 1711-1712 des moyens d'accroître encore la proportion d'assesseurs chevaleresques au $\mathrm{TCI}^{38}$. Plus généralement, on observe alors un accroissement notable de la part de la noblesse parmi les assesseurs nommés par les princes-électeurs et les Cercles d'Empire, ce qui forme un contraste marqué avec le XVIe siècle. Le Cercle de Souabe ne présenta que des juristes bourgeois pour le poste d'assesseur protestant qui lui revenait jusqu'en 1699 et au XVIIIe siècle en revanche uniquement des nobles ; pour son poste catholique, c'est même depuis 1687 que seuls des aristocrates furent proposés ${ }^{39}$.

Dans ce cas il s'agissait toutefois avant tout d'anoblis récents ou de nobles médiats et non de membres de la chevalerie immédiate, mais cette conversion nobiliaire du TCI ne manqua pas d'avoir un impact pour la chevalerie. Depuis le début du XVIIIe siècle ou, pour être plus précis, depuis la réouverture du tribunal, après plusieurs années de paralysie pendant la guerre de Succession d'Espagne, le resserrement des relations entre l'institution qui avait désormais son siège à Wetzlar et la chevalerie immédiate d'Empire avait également pris une autre direction. Parmi les assesseurs, solliciter son intégration à la chevalerie d'Empire était en effet devenu une véritable «mode $»^{40}$. Ces aspirations arrivèrent à point nommé pour la chevalerie immédiate qui avait connu une telle hémorragie de ses membres au XVIIe siècle et en particulier pendant la guerre de Trente $\mathrm{Ans}^{41}$ qu'elle avait dû se résoudre dans les dernières décennies du siècle à envisager l'idée d'accepter dans ses rangs de nouvelles familles, de noblesse ancienne ou plus récente ${ }^{42}$. On sait que la «mode» est toujours également l'expression d'une obligation de tenir son rang et d'une situation de concurrence sociale; le groupe des juges de Wetzlar en est bien souvent la démonstration. Ainsi, lorsque l'assesseur Beaurieux ${ }^{43}$, présenté par l'électeur de Mayence, fit en 1737 acte de candidature pour être reçu au sein du canton du Rhin-moyen, il argua du fait que certains de ses collègues avaient déjà eu la grâce et l'honneur d'être admis comme membres de cet illustre corps pour demander à bénéficier du même bienfait ${ }^{44}$. De même, Ferdinand Heinrich von Dünnwald ${ }^{45}$, qui avait servi l'électorat de Mayence et avait été présenté par la Bavière, exprima en 1745 l'espoir « qu'à l'instar d'autres seigneurs assesseurs de l'empereur et du tribunal de la Chambre impériale la réception me soit accordée à moi aussi $»^{46}$. Speckman, un assesseur présenté par la Bohême, fit même en 1746 nommément référence à ses collègues Beaurieux et Ulmenstein, auxquels il ne voulait céder en rien ${ }^{47}$. Bref, dans le petit monde de Wetzlar il était de plus en plus de bon ton d'appartenir à la chevalerie d'Empire et chacun mettait tout son orgueil à renforcer encore la haute idée qu'il avait de lui-même en se plaçant, par l'admission dans la chevalerie, dans une relation immédiate à l'empereur et en devenant d'une certaine manière le pair des princes de l'Empire. Cette tendance a sans doute été encore renforcée par les nombreux liens familiaux entre les assesseurs : le gendre et sa femme ne voulaient naturellement pas rester un cran en-dessous du beau-père ${ }^{48}$. Reste à 
déterminer si dans cette transhumance manifeste des assesseurs vers le statut de chevalier d'Empire la personnalité du premier Grand-juge issu des rangs de la chevalerie, le baron von Ingelheim ${ }^{49}$, a pu jouer un rôle, en aplanissant par exemple certaines difficultés.

11 La chevalerie d'Empire avait une véritable obsession de son exclusivisme social et pourtant - même si dans certains cas son cercle souabe a fait quelques difficultés pour des raisons formelles ${ }^{50}$ - elle n'a jamais fermé sa porte, autant que je sache, aux demandes d'admission émises par les assesseurs du TCI ${ }^{51}$. L'inverse aurait d'ailleurs été peu compréhensible, compte tenu des intérêts bien compris de la corporation. Les impétrants - décomplexés en outre par la science camérale, qui leur accordait la qualité de nobles de naissance quelle que soit leur origine réelle ${ }^{52}$ - savaient quant à eux comment s'y prendre pour faire savoir bien haut les services, réels ou supposés, qu'ils avaient rendus à la chevalerie dans leurs activités au sein du tribunal. Pütz, assesseur présenté par le Cercle d'Empire du Haut-Rhin, insista par exemple dans sa demande d'admission de 1744 sur le fait que depuis qu'il appartenait au TCI, il avait abondamment contribué à promouvoir les intérêts de la chevalerie ${ }^{53}$. On retrouve une argumentation comparable dans la demande rédigée en 1793 par Fahnenberg, un caméraliste renommé, pour être reçu dans le canton de Rhönwerra ${ }^{54}$. D'autres, sans doute moins bien placés pour se targuer de leurs mérites, faisaient plutôt porter leur argumentation sur l'avenir ${ }^{55}$. L'assesseur saxon Autenried fit ainsi savoir en 1791 au canton d'Odenwald : «Si je suis jamais en état de rendre à la haute chevalerie d'Empire et singulièrement au canton dont vous avez la charge quelque service utile ou agréable, j'en ferai mon affaire personnelle la plus précieuse et la plus plaisante ${ }^{56}$.

La chevalerie d'Empire s'empressait de souligner expressément les mérites passés de l'impétrant - soit, concrètement, l'aide apportée au cours de procès de certains chevaliers ou de cantons tout entiers ${ }^{57}$ - afin d'initier ou d'accélérer le processus de réception de l'assesseur en question ${ }^{58}$. Le canton franconien de Gebirg, en 1791, ne se trompait pas lorsqu'il faisait remarquer «que les demandes de ce type présentées par de tels impétrants auprès de la chevalerie immédiate d'Empire ont depuis toujours été accueillies avec une singulière bonne volonté $\aleph^{59}$. Il en est de même du jugement rétrospectif du canton d'Ortenau en février 1804 : il voit juste en estimant que l'on avait "depuis toujours immensément privilégié, pour des raisons bien connues, l'admission de messieurs les assesseurs du tribunal de la Chambre [...] au sein de la corporation des chevaliers d'Empire $»^{60}$. Les correspondances échangées entre les cantons et les cercles (au moins depuis le règlement sur les admissions de 1727, il fallait l'accord des trois cercles pour recevoir de nouveaux membres ${ }^{61}$ ) ne faisaient pas mystère des motivations utilitaristes qui animaient les chevaliers : le canton du Rhin-moyen signala ainsi en 1716, au moment de la réception de Geismar ${ }^{62}$, comme en 1728 à l'occasion de la candidature de l'assesseur Franz, que l'ensemble du corps chevaleresque serait bien inspiré d'accorder la plus grande importance à la présence au sein des tribunaux d'Empire de membres de sa corporation ${ }^{63}$. En 1760, le même canton mit les points sur les $\mathrm{i}$ en donnant son avis favorable à l'admission de Cramer von Clauspruch : selon ce texte, l'impétrant n'avait pas seulement, dans ses écrits, «traité de nombreux thèmes chevaleresques complexes dans un sens favorable au corps des chevaliers d'Empire "; il avait aussi "contribué à faire émerger une interprétation juridique » dans un grand nombre de procès de la chevalerie et il saurait à n'en pas douter donner à l'avenir « un coup de pouce conforme à la justice » aux affaires de la corporation ${ }^{64}$. On voit donc bien ce qui animait les chevaliers : il s'agissait de s'assurer un lobby puissant au TCI et, sans 
éliminer totalement l'indépendance dont les règlements faisaient obligation aux assesseurs, d'y faire du moins quelques brèches et d'en arriver à une situation où lors des nombreux procès impliquant la chevalerie d'Empire, une partie au moins de ceux qui devaient les juger pourraient être comptés comme un parti favorable, en raison des liens qui les unissaient à la corporation. Bien sûr, dans le même temps, la chevalerie s'indignait officiellement de se voir imputer de telles intentions, en rétorquant par exemple que les assesseurs étaient justement dispensés de prêter serment sur les constitutions chevaleresques ${ }^{65}$.

13 Cet intérêt particulier a très vite mis à mal les conditions d'admission dans la chevalerie - alors que pour tout autre qu'un assesseur elles étaient extrêmement sévères, si bien que par exemple les princes d'Empire s'y cassaient généralement les dents $^{66}$. Chaque canton a toujours renoncé ${ }^{67}$, à la demande de l'impétrant ${ }^{68}$ ou de son propre mouvement ${ }^{69}$, aux redevances qui étaient normalement dues - une somme de 300 florins versée à la caisse chevaleresque pour les nobles d'ancienne extraction. Ceux qui veillèrent le plus à ce que même les assesseurs du TCI s'acquittent correctement de leurs obligations financières furent sans aucun doute les chevaliers souabes. Le tribunal de Wetzlar ne jouaient en effet pas pour eux un rôle aussi important que pour leurs congénères de Franconie et du Rhin et ils eurent pour cette raison d'assez nombreux différends avec les deux autres cercles ${ }^{70}$. Mais on pouvait bien sûr passer outre l'opposition de la Souabe, comme le montre l'exemple d'Ortmann, un assesseur proposé par l'Autriche : il avait d'abord consenti à verser les redevances habituelles, mais après sa réception il avait demandé à être dispensé de cette obligation en arguant des précédents. Le statut d'Heilbronn de 1750, édicté avant tout sous l'influence de la Souabe, avait bien interdit à chacun des cercles et des cantons de consentir spontanément à une ristourne, mais en 1754 l'assemblée générale de la chevalerie avait quant à elle laissé à chacun le soin d'en décider, si bien que le canton du Rhin-moyen (cercle rhénan) parvint à imposer après coup la réception gratuite de l'assesseur Ortmann $^{71}$. Les chevaliers firent preuve d'une prévenance encore plus libérale: on dispensa les impétrants anoblis d'être explicitement accueillis comme nobles de frâiche date $^{72}$, car cela les aurait rendu passibles d'un versement beaucoup plus important et les aurait en outre exclus de toutes les fonctions dirigeantes au sein d'un canton. Lorsque l'assesseur n'avait pas encore acquis le bien chevaleresque immédiat, dont la possession était obligatoire pour être admis, on se contentait de fermer les yeux et on se satisfaisait d'une promesse - purement formelle, dans la plupart des cas - d'en acheter bientôt un $^{73}$. Les cas où les assesseurs, avant de faire leur demande, avaient effectivement acheté un petit domaine chevaleresque, ne furent pas très nombreux ${ }^{74}$; la plupart du temps, on recourait à un artifice argumentatif permis par le statut d'Heilbronn de 1750 : la fonction d'assesseur au TCI était réputée qualifier à elle seule pour l'appartenance à la chevalerie d'Empire. En tout cas, les juges de Wetzlar - à la différence de ce que l'on constate pour les autres réceptions - n'ont jamais été considérés avant tout comme des instruments pour pallier à l'érosion qui menaçait les portions de territoire relevant de la corporation chevaleresque. Les cantons et les cercles firent preuve de la même mansuétude lorsque les arbres généalogiques des candidats n'étaient pas à la hauteur des exigences ${ }^{75}$ ou même manquaient au dossier. Dans ce domaine aussi, c'est le cercle souabe qui s'attacha à faire respecter le plus strictement possible les règlements sur la réception de nouveaux membres, s'opposant au laxisme des deux autres cercles ${ }^{76}$ et s'attirant ainsi les foudres non seulement des assesseurs concernés ${ }^{77}$ mais aussi des chevaliers franconiens et rhénans, même si ceux- 
ci veillaient naturellement à ce que les candidats présentent un minimum de valeur nobiliaire. Bien sûr, les chevaliers ressentaient une grande satisfaction lorsque les doutes sur le sang bleu d'un impétrant issu du TCI étaient levés par des lettres de noblesse hâtivement obtenues auprès de l'empereur, comme ce fut par exemple le cas en 1764 pour Papius, assesseur présenté par le Cercle d'Empire bourguignon ${ }^{78}$. Au total, il est certain que les cercles et les cantons de Franconie et de Rhénanie se sont efforcés de donner la priorité absolue à la réception d'assesseurs du tribunal de la Chambre impériale et d'y procéder le plus rapidement possible, dans le but de ne pas mécontenter ce genre de candidats et d'éviter qu'ils n'en "conçoivent du dégoût ». C'est en pensant aux juges de Wetzlar que les chevaliers franconiens et rhénans sont par exemple montés au créneau en 1729 contre une manœuvre des Souabes pour ne plus traiter des réceptions (lesquelles nécessitaient l'approbation des trois cercles), qu'au cours des assemblées générales de la chevalerie (Generalkorrespondenztag) qui n'avaient lieu communément que tous les trois ans ou moins fréquemment encore ${ }^{79}$. Les procédures s'étalant pour des raisons techniques sur une dizaine d'années, comme celle de Dünnwald (1745-1755), furent tout à fait exceptionnelles.

La conjonction de ces deux évolutions - l'appétence croissante des chevaliers d'Empire anciens pour les carrières du TCI et la tendance des autres assesseurs à briguer l'admission dans la corporation - conféra au tribunal de Wetzlar un caractère chevaleresque si marqué qu'il finit par devenir un objet de scandale pour plus d'un prince et d'un auteur ${ }^{80}$. Il ne faut pas oublier en effet que même les positions les plus prestigieuses au sein du tribunal, celles de Grand-juge et de président, furent de plus en plus souvent occupées par des chevaliers d'Empire ${ }^{81}$ ou des hommes qui, à l'instar du comte médiat von Spaur, sollicitaient leur admission dans la chevalerie d'Empire immédiatement après avoir accédé à leur dignité ${ }^{82}$. Considérons la situation, au hasard, en 1750: l'un des deux présidents (Groschlag ${ }^{83}$ ) et pas moins de onze des seize assesseurs en poste sont membres de la chevalerie d'Empire, ou viennent de le devenir, ou ont posé leur candidature pour le devenir, et la proportion s'accroît encore dans les années suivantes ${ }^{84}$. En 1750, quelques principautés protestantes, sous la houlette du Wurtemberg, entreprirent d'œuvrer à la Diète à une réduction de l'influence de la chevalerie sur les tribunaux d'Empire ${ }^{85}$. Cette tentative visait tout particulièrement les connexions personnelles tissées entre la chevalerie d'Empire et le TCI. Toujours à la Diète de Ratisbonne, le Palatinat électoral introduisit en 1766 une proposition - dont on ignore l'issue - pour que «l'on veille à ce que seules un certain nombre de personnes issues de la chevalerie immédiate d'Empire soient admises aux postes d'assesseurs, et que par ailleurs parmi les autres assesseurs aucun ne soit, pendant qu'il est en charge [...], admis dans l'une des chevaleries en Franconie, Souabe et Rhénanie $»^{86}$. Lors de la dernière inspection du tribunal, il y eut parmi les délégués des princes un fort mouvement en faveur d'une délibération spécifiquement consacrée à cette question des liens personnels entre chevalerie et TCI; mais sans résultat ${ }^{87}$. Ce contexte politique, marqué par la volonté de réduire l'influence de la chevalerie d'Empire sur le tribunal de Wetzlar, contribue également à expliquer la confrontation bien inutile qu'entamèrent quelques princes d'Empire dans les années 1770-1771 sur la question de savoir si l'exercice d'une présidence au TCI était compatible avec la dignité de directeur d'un canton de chevalerie ${ }^{88}$. Cette attaque des états d'Empire contre la chevalerie immédiate put d'ailleurs être repoussée grâce à une argumentation particulièrement habile qui fit valoir que l'empereur avait tout intérêt à se savoir soutenu par certains membres du 
TCI, que les chevaliers d'Empire étaient les plus sûrs de ses alliés, que l'objectif des princes était en fait de limiter le libre exercice de son droit de nomination, etc.

En dépit de ces signes évidents d'un rapprochement entre la corporation chevaleresque et le tribunal depuis la fin du XVIIe siècle, les cercles et les cantons refusèrent longtemps tout commencement de discussion sur une possible contribution financière au fonctionnement du TCI. Depuis que la chevalerie d'Empire, dans les années 1540, avait définitivement obtenu d'être exemptée de toute charge commune, elle n'avait du même coup plus jamais été sollicitée pour le financement de la Chambre et il est remarquable qu'il fallut attendre un bon siècle avant que la question soit de nouveau évoquée. Lorsqu'elle le fut, la réaction des chevaliers fut toujours extrêmement négative: derrière l'hypothèse d'une contribution au TCI ils voyaient en effet se profiler le danger d'être, plus généralement, soumis aux impôts d'Empire. Par bonheur pour eux, ils trouvèrent dans leur acharnement à défendre leur exemption un avocat de poids en la personne de l'empereur, qui ne voulait pas voir se tarir la source financière que représentaient les «subsides caritatifs", versés directement au monarque par les chevaliers d'Empire. Par chance aussi, plusieurs princes craignaient que les chevaliers, s'ils étaient mis au nombre de ceux qui payaient pour le TCI, puissent se mettre à désirer obtenir en contrepartie toutes les prérogatives des états d'Empire. La conjonction de ces deux arrière-pensées fit que, lors de la Diète de Ratisbonne en 1653-1654, les propositions visant à faire des chevaliers des contributeurs au fonctionnement du tribunal de la Chambre furent rapidement remisées dans un tiroir ${ }^{89}$. La chevalerie avait mobilisé ses consultants habiles à manier la plume - comme Kreidemann et Knipschild, juristes à Eßlingen - pour élaborer de savantes dissertations donnant un fondement juridique à leur droit de refuser de verser le moindre sou. La cour de Vienne appréciait le renfort de ces francs-tireurs lorsqu'elle devait repousser les récriminations de certains états d'Empire envers les privilèges et libertés accordés par les empereurs à la chevalerie d'Empire ${ }^{90}$. Lorsqu'en 1720-1723 les débats reprirent sur le financement du tribunal ${ }^{91}$, les cantons de chevalerie actionnèrent à nouveau tous les leviers permettant de sauvegarder leur exemption, avec l'aide de l'empereur et de l'archevêque-électeur de Mayence ${ }^{92}$. Un demi-siècle plus tard, alors que se déroulait la dernière inspection du tribunal, la question d'une éventuelle participation financière de la chevalerie d'Empire au fonctionnement du TCI revint encore une fois sur le tapis ${ }^{93}$. Cette fois, c'est le tribunal lui-même qui suggéra que la chevalerie puisse obtenir le droit de présenter deux assesseurs en contrepartie d'une cotisation (proposition émise d'ailleurs après que la majorité des membres de la commission d'inspection se fut prononcée en faveur d'une mise à contribution des chevaliers), mais sans exprimer la volonté de leur concéder la moindre compensation. La nervosité excessive avec laquelle la chevalerie réagit est une nouvelle illustration de son intransigeance sur ce point: réunie en octobre 1772 en assemblée générale à Nuremberg, elle clama que l'empereur verrait bien vite que l'obligation pour la chevalerie de faire ce paiement une fois établie (même assortie du droit de présentation) ouvrirait bientôt la voie à bien d'autres exigences financières. Ce fardeau ne pourrait qu'affaiblir sa capacité à se vouer uniquement au bien de l'empereur dans toutes les occasions qui se présentaient. L'assemblée priait donc l'empereur - sousentendu : dans son propre intérêt - de tout faire, au milieu de cette crise extrêmement préoccupante, pour que les avis du tribunal de Wetzlar et de la commission d'inspection soient jetés aux oubliettes ${ }^{94}$. Bien que les interactions personnelles entre le TCI et la chevalerie n'aient cessé depuis plusieurs décennies de se densifier, cette 
dernière n'était donc toujours pas prête à sauter le pas lui permettant de se lier plus étroitement encore avec les juges de Wetzlar pour lutter ensemble au service du maintien des institutions de l'Empire. L'engagement financier de la chevalerie en faveur du tribunal aurait certainement constitué pour elle une charge préjudiciable, mais en contrepartie le corps chevaleresque y aurait sans aucun doute gagné une implantation plus grande dans le système impérial et donc une possibilité de se dégager de la relation trop exclusive qui le liait à l'empereur. C'est dans cet horizon que s'inscrivaient aussi les propositions de réforme que fit, pour poursuivre ce débat sur la participation financière de la chevalerie au tribunal de Wetzlar, le syndic du cercle rhénan, Tabor - sans soulever d'ailleurs un grand écho ${ }^{95}$. Ce projet visait en effet à donner à la chevalerie une forme d'organisation qui en aurait fait quasiment un État territorial ${ }^{96}$ et qui aurait ouvert la voie à son accession au statut d'état d'Empire, contribuant ainsi à l'ancrer dans toutes les institutions impériales. Car le lien de dépendance exclusive envers l'empereur de la Maison de Habsbourg-Lorraine ne pouvait que s'avérer néfaste à partir du moment où ce monarque perdait progressivement tout intérêt envers l'Empire, cherchait de plus en plus à s'en détacher, et l'abandonnait à son propre sort - et avec lui la chevalerie, désormais sans aucun soutien, alors que s'annonçait la lutte pour sa propre existence. L'empereur, parce qu'il tournait justement la page de l'Empire, devenait ainsi depuis la fin du XVIIIe siècle un danger indirect pour la survie de la chevalerie, aux côtés de la menace directe que représentait l'hostilité des princes. Mais la plupart des chevaliers ne se sont aperçus que trop tard de cette évolution. Une étude de Gerhard Pfeiffer a montré il y a quelques années que certains des cantons, ainsi qu'un des plus importants alliés de la chevalerie d'Empire, l'électorat de Mayence, avaient certes pris conscience de ce risque bien avant que Vienne ne manifeste son indifférence envers le sort de l'Empire. Depuis le milieu du XVIIe siècle ils avaient par intervalles très irréguliers (dans les années suivant 1645 , en 1664, en 1685-1688, en 1771-1772) essayé d'obtenir - à l'instar des comtes et des villes d'Empire, mais sans le même succès - le statut d'état d'Empire pour la chevalerie immédiate. Lors de la grande crise de confiance entre la Maison d'Autriche et les chevaliers, aux lendemains de 1648, ils échafaudèrent même brièvement le projet politique de faire de l'archichancelier d'Empire (l'électeur de Mayence) une sorte de puissance tutélaire de la chevalerie ${ }^{97}$. Mais deux facteurs déterminants s'opposèrent à ces tentatives: l'incapacité constante des trois cercles à élaborer une position commune sur ce sujet ${ }^{98}$; et la résistance opiniâtre de l'empereur, des états d'Empire en général et particulièrement des villes d'Empire, qui craignaient de devoir rétrograder dans l'ordre des préséances ${ }^{99}$.

Il n'est guère surprenant que les propositions visant à introduire une contribution financière des chevaliers d'Empire au fonctionnement du tribunal de Wetzlar aient à nouveau fait leur apparition, dans les débats politiques comme dans les publications, à l'époque de la Ligue des princes - c'est-à-dire à un moment où tous avaient pu constater que la relation de Joseph II à l'Empire n'allait pas sans difficultés, et où le TCI, justement, redevenait une sorte de symbole de la pérennité et de l'adaptation des institutions d'Empire au milieu des vastes projets de réforme qui le concernaient. Ainsi, pendant toute l'année 1789 , un écrit intitulé "Opus posthumum ", qui s'était mis à circuler à Ratisbonne, nourrit les conversations et les correspondances des cercles et des cantons de la chevalerie d'Empire. Son auteur anonyme essayait longuement de donner consistance à sa proposition : amener la chevalerie à assurer le financement de quatre assesseurs et lui concéder en contrepartie le statut d'état d'Empire et la 
participation aux Cercles d'Empire. Les milieux qui n'étaient pas trop éloignés de la chevalerie étaient eux aussi de plus en plus conscients de la nécessité d'asseoir la relation entre la chevalerie et le TCI sur des fondements nouveaux; c'est aussi en 1789 que Kerner formula sa célèbre question : n'y a-t-il pas davantage qu'une anomalie dans le fait que les chevaliers immédiats ne versent pas d'argent pour le tribunal de Wetzlar alors qu'un tiers des procès devant cette chambre les concerne, et dans la présence de chevaliers au sommet de la hiérarchie des juges alors que dans la lettre des règlements ils en sont exclus ${ }^{100}$ ? Reste que même en 1789 la plupart des cantons continuaient à se montrer sceptiques envers la proposition de l'« Opus posthumum». Cependant, et pour la première fois, l'un d'entre eux - le canton du Rhin-moyen, comme on l'a vu au tout début de cet article - parvint à réserver à cette idée un accueil moins glacial ${ }^{101}$. Inutile de dire que les événements qui se précipitèrent dans les années suivantes n'offrirent pas à ce projet le terreau le plus favorable à sa germination: la Révolution française amena la guerre, l'Empire commença vite à se déliter, la médiatisation de la chevalerie d'Empire débuta en Franconie peu après 1792, en Souabe en 1805. Mais dans le même temps, autour de 1800, tandis que le vent de l'histoire ébranlait les institutions traditionnelles du vieil Empire et que plus personne n'aurait misé sur leur survie, les assesseurs de Wetzlar persistaient - de manière démonstrative, serait-on tenté de dire - à solliciter leur admission dans la chevalerie d'Empire, et à y être reçus, comme Linden en 1800 et Branca en 1804. Les cantons qui existaient encore mirent, dans le cas de Branca, issu d'une famille du Nord de l'Italie, encore une fois sous le boisseau les sérieux doutes qui pesaient sur la qualité ou même la réalité de sa noblesse, afin de pouvoir s'assurer le soutien d'un partisan supplémentaire de «la pérennité des institutions chevaleresques conformément aux constitutions de l'Empire $»^{102}$.

J'en arrive à la conclusion et souhaite rassembler les résultats obtenus en trois points :

18 1. Alors que le nouveau tribunal central, porté avant tout par l'initiative des princes de l'Empire, n'avait soulevé dans les premières décennies suivant sa mise en place qu'un scepticisme marqué et une attitude de refus de la part de la chevalerie d'Empire, qui ne montra au départ aucune disposition à s'accommoder de son existence, cette relation se transforma diamétralement à partir de la première moitié du XVIIe siècle: une convergence croissante s'établit entre la Chambre impériale et la chevalerie en vertu des liens personnels de plus en plus étroits entre les deux institutions.

19 2. Cette intensité grandissante des connexions entrecroisées ne suffit pourtant pas à détourner la chevalerie de sa dépendance exclusive envers le soutien de l'empereur et à s'ancrer davantage dans le système impérial en acquérant le statut d'état d'Empire et en contribuant au financement des juges de Wetzlar.

20 3. Le tribunal de la Chambre impériale et la chevalerie d'Empire, une institution et une organisation sur lesquelles pleuvaient dès le XVIIIe siècle les sarcasmes visant l'absence (supposée) d'efficacité et de rendement pour la première, l'archaïsme des structures pour la seconde, peuvent donner l'image d'idoles poussiéreuses d'une constitution vieillie, jurant dans le décor des temps nouveaux. Elles méritent pourtant, à mon sens, autre chose que le mépris car ce sont elles - et non l'empereur, les princes et le Conseil impérial aulique - qui sont restées loyales jusqu'au bout à l'idée du Sacrum Imperium. Mais ce jugement globalement négatif qui était alors celui de l'opinion publique ${ }^{103}$ les empêcha en définitive de ressusciter lors du congrès de Vienne - sous la forme renouvelée d'un tribunal suprême de l'Empire ${ }^{104}$ et d'une véritable organisation corporative de la chevalerie. Les États, grands ou moyens, donnaient désormais le ton 
dans la Confédération Germanique et ils n'avaient aucun intérêt à maintenir des gardefous de l'unité impériale ou des organismes qui faisaient obstacle à leur aspiration vers une souveraineté sans partage ${ }^{105}$.

\section{BIBLIOGRAPHIE}

\section{Litérature secondaire}

Bader, K. S. (1950) : Der deutsche Südwesten in seiner geschichtlichen Entwicklung, Stuttgart.

Blickle, P. (1976) : « Gemeiner Pfennig und Obrigkeit (1495) », Vierteljahrschrift für Sozial- und Wirtschaftsgeschichte, 63, p. 180-193.

Bloem, T. (1969) : Verfassungsgerichtliche Probleme von 1495-1806, thèse de droit, Kiel.

Diel, K. (1957) : « Groschlag in Wetzlar », in :Festschrift zum 70. Geburtstag für Adolph Frhr. von Gemmingen-Hornberg, Francfort, p. 54-62.

Diestelkamp, B. (1976) : « Das Reichskammergericht im Rechtsleben des 16. Jahrhunderts », in :Rechtsgeschichte als Kulturgeschichte (Festschrift für Adalbert Erler), Aachen, p. 435-480.

Duchhardt, H. (1970) : « Reichskammerrichter Franz Adolf Dietrich von Ingelheim (1659/1730-1742)», Nassauische Annalen, 81, p. 173-202.

Duchhardt, H. (1977a) : « Die kurmainzischen Reichskammergerichtsassessoren », Zeitschrift der Savigny-Stiftung für Rechtsgeschichte, Germanistische Abteilung, 94, p. 89-128.

Duchhardt, H. (1977b) : « Mainzer Professoren am Reichskammergericht » in : Tradition und Gegenwart, vol. 1 : Aus der Zeit der kurfürstlichen Universität, éd. par H. Weber, Wiesbaden, p. 100-117.

Fellner, R. (1905) : Die fränkische Ritterschaft von 1495-1524, Berlin.

Hellstern, G. (1971) : Der Ritterkanton Neckar-Schwarzwald 1560-1805, Tübingen.

Hofmann, H. H. (1962) : Adelige Herrschaft und souveräner Staat, Munich.

Huschke, W. (1974) : « Johann Heinrich Tabor (1728-1802), reichsritterschaftlicher Syndikus zu Friedberg ", Archiv für hessische Geschichte und Altertumskunde, NF 32, p. 283-326.

Jahns, S. (1976) : Frankfurt, Reformation und Schmalkaldischer Bund, Francfort.

Kirchner, H. (1968) : « Das Ringen um ein Bundesgericht für den Deutschen Bund », in :Ehrengabe für Bruno Heusinger, p. 19-33.

Mager, W. (1974) : « Das Problem der landständischen Verfassungen auf dem Wiener Kongreß 1814/15 », Historische Zeitschrift, 217, p. 197-215.

Mauchenheim gt. Bechtolsheim, H. Freiherr von (1972) : Des Heiligen Römischen Reichs unmittelbarfreie Ritterschaft zu Franken Ort Steigerwald im 17. und 18. Jahrhundert, Wurzbourg.

Mielke, H.-P. (1977) : Die Niederadligen von Hattstein, ihre politische Rolle und soziale Stellung, Wiesbaden. 
Pfeiffer, G. (1962) : « Studien zur Geschichte der fränkischen Reichsritterschaft », Jahrbuch für fränkische Landesforschung, 22, p. 173-280.

Praetorius, O. (1937) : «Juristen-Sippen am alten Reichskammergericht », Familiengeschichtliche Blätter/ Deutscher Herold, 35, col. 67-78.

Press, V. (1974) : « Die Ritterschaft im Kraichgau zwischen Reich und Territorium 1500-1623 », Zeitschrift für die Geschichte des Oberrheins, 121, p. 35-98.

Press, V. (1976a) : Kaiser Karl V., König Ferdinand und die Entstehung der Reichsritterschaft, Wiesbaden.

Press, V. (1976b) : « Die Reichsritterschaft im Reich der frühen Neuzeit », Nassauische Annalen, 87, p. 101-122.

Riedenauer, E. (1969) : « Kontinuität und Fluktuation im Mitgliederstand der fränkischen Reichsritterschaft » in : Gesellschaft und Herrschaft (Festgabe für Karl Bosl), Munich, p. 87-152.

Roth von Schreckenstein, K. H. (1871) : Geschichte der ehemaligen Reichsritterschaft in Schwaben, Franken und am Rheinstrome, vol. 2, Fribourg et Tübingen.

Smend, R. (1911) : Das Reichskammergericht. Geschichte und Verfassung, Weimar.

Stetten, W. von (1973) : Die Rechtsstellung der unmittelbaren freien Reichsritterschaft, ihre Mediatisierung und ihre Stellung in den neuen Landen. Dargestellt am fränkischen Kanton Odenwald, Schwäbisch Hall.

Ulmann, H. (1872) : Franz von Sickingen, Leipzig.

Weitzel, J. (1976) : Der Kampf um die Appellation ans Reichskammergericht, Cologne et Vienne.

\section{Sources}

Annotata de personis iudicii camerae imperialis..., Ingolstadt, 1557.

Burgermeister, J. S. (1707) : Reichsritterschaftliches Corpus Juris, Ulm.

Burgermeister, J. S. (1721) : Codex diplomaticus equestris, Ulm.

Cramer, J. U. von (1766) : Observationum juris universi ex praxi recentiori supremorum Imperii tribunalium haustarum..., t. IV, Ulm.

Decker-Hauff, H. (éd.) (1973) : Die Chronik der Grafen von Zimmern, Darmstadt.

Deutsche Reichstagsakten, Jüngere Reihe, vol. 2, présenté par Adolf Wrede, Gotha, 1896.

Deutsche Reichtagsakten, Jüngere Reihe, vol. 3, présenté par Adolf Wrede, Gotha, 1901.

Fahnenberg, E. J. K. von (1792) : Litteratur des Kaiserlichen Reichskammergerichts, Wetzlar.

Harpprecht, S. C. (1727) : Sacri Romani Imperii liberae et immediatae nobilitatis prae civitatibus imperialibus jus sessionis et praecedentiae..., Hambourg et Leipzig.

Herrmann, F. (1792) : Juristische Abhandlung von der Aufnahme in die Reichsritterschaftliche Genossenschafft..., Mayence.

Kerner, J. G. (1789) : Staatsrecht der unmittelbaren freyen Reichsritterschaft in Schwaben, Franken und am Rhein, Lemgo.

Kopp, J. A. (1728) : Tractatus juris publici de insigni differentia inter S.R.I. Comites et nobiles immediatos..., $2^{\mathrm{e}}$ éd., Strasbourg. 
Laufs, A. (éd.) (1976) : Die Reichskammergerichtsordnung von 1555, Cologne et Vienne.

Ludolf, G. M. von (1765) : Catalogus Personarum Collegii Cameralis ab anno MDCLIV..., s.l.

Mader, J. (éd.) (1788) : Reichsritterschaftliches Magazin, livraison 10, Francfort et Leipzig.

Moser, J. J. (1772) : Vermischte Nachrichten von reichsritterschaftlichen Sachen, livraison 2, Nuremberg.

Moser, J. J. (1774) : Neues Teutsches Staatsrecht, vol. 8/2, Francfort et Leipzig.

Moser, J. J. (1775a) : Beyträge zu Reichsritterschaftlichen Sachen, livraison 1, Ulm, Francfort et Leipzig.

Moser, J. J. (1775b) : Neueste Geschichte der unmittelbaren Reichsritterschaft, partie 1, Francfort et Leipzig.

Schade, O. (1863) : Satiren und Pasquille aus der Reformationszeit, $2^{\mathrm{e}}$ éd., vol. 2, Hanovre.

Schmauss, J. J. / Senckenberg, H. C. von (1747) : Neue und vollständigere Sammlung der

Reichsabschiede..., vol. 2.

Schmidt-Scharff, W. (1934) : « Die Matrikel der Praktikanten am Reichskammergericht in

Wetzlar 1693-1806 », Archiv für Sippenforschung, 11, p. 299-317.

Tafinger, F. W. (1764) : An assessorem Camerae Imperialis praesentandi jure gaudeat S.R.G.I. nobilitas immediata..., Tübingen.

Vahlkampf, J. A. (1806) : Reichskammergerichtliche Miscellen, vol. 2, Gießen et Wetzlar.

\section{NOTES}

1. Cité par Ulmann (1872), p. 299, note 2. Il est également intéressant de voir le jugement sans appel du tribunal de la Chambre impériale que les publicistes du temps attribuent à Sickingen. Dans le Dialogus so Franciscus von Sickingen vor des Himels Pforten mit Sant Peter und dem Ritter Sant Jörgen gehalten zuvor und ehe dann er ingelasten ist worden, l'auteur anonyme lui fait dire la chose suivante au sujet de cette institution d'Empire : « [le TCI] est une chose comme ça : celui qui en a fini avec un tribunal inférieur comme si c'était le purgatoire, se retrouve alors confronté à l'enfer. Car je pense en vérité qu'aucune âme ne peut être davantage tourmentée en enfer par les diables que quand un pauvre homme est livré aux procureurs, avocats et autre engeance rouillée, car alors ce ne sont qu'action, exception, réplique, duplique, triplique et quadriplique, reports, peremptoriales ferie in novis prefaxis et ordinariis, si bien qu'on n'en a jamais terminé : ils n'en finiront pas avant de vous avoir dévoré chair et sang. Si sur cent il en est un qui obtient enfin une sentence définitive, il lui faut encore aller chercher l'exécution de sa sentence chez le maître queux d'Ochsenstein. Il était si plein de force que quand il préparait une soupe pour quelqu'un il lui faisait dévaler les escaliers. La conséquence d'un tel déni de justice est que là où un pauvre homme n'est pas en capacité d'attendre la fin de toutes ces pompes interminables et insupportables, alors il entreprend une faide, des hostilités ou une guerre... » (Schade [1863], p. 55).

2. Le canton du Rhin-Moyen au canton du Rhin-inférieur, 20 nov. 1789 (LHAKo 53B 703).

3. Burgermeister (1721), vol. 1, p. 924.

4. Voir Hellstern (1971), p. 41-42. Le lien inconditionnel établi avec l'empereur est attesté par d'innombrables sources, par exemple l'article 1 de l'ordonnance chevaleresque souabe de 1561 (Burgermeister [1721], vol. 1, p. 170), le projet d'ordonnance chevaleresque souabe de 1653 (ibid., p. 187), le titre III de l'ordonnance chevaleresque franconienne de 1718 (Stetten [1973], 
p. 267-268), etc. - Les empereurs quant à eux, sur la base de cette étroite alliance, ont prétendu exercer dans la pratique politique une sorte de droit de surveillance sur les cantons et les cercles de l'organisation chevaleresque (exemple dans Hellstern [1971], p. 51). Le chef de l'Empire s'est fait l'avocat des intérêts chevaleresques lors de l'élaboration des lois d'Empire (ibid., p. 52) et, jusque dans les premières décennies du XVIIIe siècle, il a œuvré en faveur du renforcement de la corporation en confirmant à de nombreuses reprises les prérogatives des chevaliers d'Empire (voir par exemple le recensement présenté dans ibid., p. 58 sq.).

5. Voir Press (1976a).

* NDT: la répartition régionale regroupant les différents territoires (états d'Empire) porte le même nom que l'organisation corporative des chevaliers d'Empire, elle aussi répartie selon trois ensembles géographiques discontinus (Kreis). Pour éviter la confusion, on a respecté tout au long $\mathrm{du}$ texte la convention suivante: les Cercles d'Empire, dans le premier cas, porteront une majuscule; les cercles des chevaliers n'en porteront pas. La distinction est d'autant plus nécessaire que les cercles de chevalerie font partie des rares organes de l'Empire à ne pas être inclus dans les Cercles.

6. Pour une "étude de cas» sur ce combat de la chevalerie afin d'obtenir l'immédiateté d'Empire, on peut recourir à Press (1974).

7. On peut du reste dire à peu près la même chose des villes libres, exclues elles aussi de la nomination et de l'entretien des juges du TCI « princier »; voir Smend (1911), p. 110 sq. et Bloem (1969), p. 32.

8. Deutsche Reichtagsakten, Jüngere Reihe, vol. $3, \mathrm{~N}^{\circ} 113$ (29 déc. 1522), en particulier p. 710 sq. Voir Roth von Schreckenstein (1871), p. 200 et Hellstern (1971), p. 20.

9. Voir par exemple Fellner (1905), p. 138 et 201, ainsi que Smend (1911), p. 109-110.

10. Voir par exemple Fellner (1905), p. 120.

11. Cet aspect s'exprime fortement, par exemple, dans Deutsche Reichstagsakten, Jüngere Reihe, vol. 2, p. 446. Sur le Gemeiner Pfennig en général, voir avant tout une référence récente : Blickle (1976), sur la politique des chevaliers à son égard voir en particulier p. 186-187 et 191, note 56.

12. Voir par exemple Fellner (1905), p. 157 sq. et passim. Cette procédure leur fut enfin accordée dans l'ordonnance sur le TCI de 1521 en vertu d'une médiation de l'empereur. Sur la genèse de cette mesure, voir Deutsche Reichstagsakten, Jüngere Reihe, vol. 2, p. 244-267.

13. Voir par exemple Smend (1911), p. 109. Dans l'« union fraternelle » de la chevalerie conclue à Landau le 13 août 1522, tout appel au TCI pour les conflits entre membres de l'union fut par principe prohibé : Ulmann (1872), p. 253.

14. Voir par exemple Press (1974), p. 49.

15. Ordonnance sur le TCI de 1521, partie IV, § 1 (Schmauss / Senckenberg [1747], vol. 2, p. 180).

16. Decker-Hauff (1973), vol. 3, p. 139.

17. Decker-Hauff (1973), vol. 2, p. 326.

18. Sur le docteur Arnold von Glauburg, patricien humaniste de Francfort, voir désormais Jahns (1976), p. 32.

19. Voir Annotata de personis iudicii camerae imperialis... . Plus généralement, sur la rapidité des rotations de personnels parmi les assesseurs du TCI, par exemple au XVIe siècle: Duchhardt (1977a), p. 118-119.

20. Un exemple, entre beaucoup d'autres, du combat de la chevalerie d'Empire contre l'appel au TCI : les articles de Schweinfurt, élaborés lors de l'assemblée de la chevalerie franconienne en 1539 et édités par Pfeiffer (1962), p. 173-280, ici p. 205.

21. Passage correspondant de l'ordonnance sur la Chambre impériale : II, § III-IV (Laufs [1976], p. 169-176). Il faudra certes encore beaucoup de temps avant que l'on dispose de données statistiques exactes sur cette abstention. Au sujet des difficultés que présentent les recherches sur l'histoire du TCI : Diestelkamp (1976). 
22. Pour autant, les corporations de chevalerie étaient parfaitement capables d'apprécier les avantages que présentaient les procédures établies pour le TCI, comme le montre par exemple la manière dont le canton de Neckar-Forêt-Noire a façonné la procédure d'exécution des sentences prononcées par le directoire du canton sur le modèle des procédures équivalentes instaurées par l'ordonnance de 1555 ; voir Hellstern (1971), p. 161.

23. Voir Stetten (1973), p. 43 (ordonnance chevaleresque franconienne de 1590).

24. Burgermeister (1721), vol. 1, p. 191-192. Quelques rares familles chevaleresques s'efforcèrent toutefois d'obtenir dès le milieu du XVIe siècle des privilèges impériaux leur permettant de relever du TCI pour le droit civil - par exemple quand elles voulaient éviter de passer devant le tribunal territorial de Wurtzbourg ou le Hofgericht de Rottweil. Voir Stetten (1973), p. 64.

25. Ainsi, le canton de Neckar-Forêt-Noire fut au sein du cercle de Souabe le seul qui maintint jusqu'en 1805 l'exercice effectif de la juridiction cantonale. Mais même là, les litiges pour lesquels cette instance était compétente furent limités en 1695 à une valeur de 200 florins ; voir Hellstern (1971), p. 157.

26. Voir Weitzel (1976), p. 233 sq.

27. Les cantons de Souabe comme celui de Neckar-Forêt-Noire, davantage tournés vers l'Autriche, avaient par exemple traditionnellement tendance à s'adresser au Conseil impérial aulique (voir Hellstern (1971), p. 159) et on a pu montrer que le canton franconien de Steigerwald suivait le même mouvement (Mauchenheim [1972], p. 430-431) alors qu'au contraire la chevalerie rhénane et une partie de la chevalerie franconienne ont pu accorder la préférence au TCI, situé plus près.

28. Kerner (1789), p. 77.

29. Il arrivait en outre que les différentes corporations chevaleresques fassent elles-mêmes parvenir leurs privilèges au tribunal et demandent un accusé de réception; voir Kerner (1789), p. 77-78.

30. Voir Hellstern (1971), p. 61-62 ; sur le contexte : Press (1974), p. 64-65. La version imprimée de la prescription impériale du 20 sept. 1591 se trouve dans Burgermeister (1707), p. 318 sq. et dans Burgermeister (1721), vol. 1, p. 276 sq. Le décret du 9 juillet 1605 est dans Burgermeister (1721), vol. 1, p. 278-279 et (1707), p. 320-321. Pour le décret du 12 janv. 1632 : Burgermeister (1707), p. 376-377 et (1721), vol. 1, p. 324-325. Voir également Moser (1775b), p. 84-85 - d'autres prescriptions du même genre, entre autres, dans Burgermeister (1721), vol. 1, p. 273 sq. (1688) et vol. 2, p. 1061-1062 (1718). Sur l'ensemble, voir Kerner (1789), vol. 3, p. 76 et 78-79.

31. Voir Kerner (1789), vol. 3, p. 81-85 ainsi que Moser (1775b), p. 430 sq.

32. Ce combat contre les juridictions des princes continua plus tard à jouer un rôle pour les chevaliers; en 1688 par exemple, le premier assesseur à être intégré dans la chevalerie immédiate, Huldreich von Eyben, dut signer un document où il s'engageait solennellement à ne jamais défendre son droit, ou accepter une sentence, auprès de tribunaux princiers (StADa F1 42/16).

33. Les plus importants de ces textes sont les «avis» des chevaliers d'Empire en 1599 (Burgermeister [1721], vol.1, p.946-959) et ceux présentés par la Franconie en 1613 (Burgermeister [1721], p. 968-989).

34. Voir Press (1974), p. 73.

35. Du côté des comtes, quelques protagonistes de ce conflit: Johann Adam Kopp ([1728], p. 510-536) et Johann Jakob Moser ([1772], p. 225-267). Voir également Duchhardt (1970), p. 181-182.

36. Voir Smend (1911), p. 260. Sur les oppositions entre comtes et chevaliers d'Empire au sujet des postes de président, voir par exemple Moser (1775b), p. 300 (protestation des comtes souabes contre la nomination comme présidents des chevaliers Dalberg et Schmidtburg) et ibid., p. 530 (protestation contre la nomination d'Ingelheim), ainsi que la lettre envoyée le 24 févr. 1724 par Ingelheim au canton du Rhin-Supérieur (StADa F2 8c), à la suite de laquelle la chevalerie 
d'Empire envoya même un ambassadeur (le baron Keller) représenter ses intérêts à Vienne (StADa F2 4/9). Cet activisme reprit en 1712 à l'occasion d'un séjour à Vienne du président SolmsLaubach - un comte d'Empire protestant: lettre de la chevalerie d'Empire à l'empereur, présentée le 18 déc. 1712 (HHStAW Reichskanzlei Reichskammergerichtsvisitationsakten 352a), disant que l'empereur devrait soutenir la chevalerie contre les prétentions indues des comtes d'Empire puisque les ordonnances sur le tribunal de la Chambre impériale de 1500 et 1555 n'avaient précisément instauré aucune différence entre les comtes et les barons.

37. Il convient en outre de mentionner qu'au XVIIIe siècle quelques anciens employés de la corporation chevaleresque ont poursuivi leur carrière au TCI, comme par exemple Speckmann qui avait été directeur de la chancellerie du canton du Rhin-inférieur (LHAKo 53B 1939) et qui dans ces fonctions avait déjà joué un rôle non négligeable lors de la nomination du chevalier d'Empire Ingelheim comme Grand-juge du TCI (Kammerrichter) en 1729-1730 (lettre du canton du Rhin-inférieur au canton du Rhin-moyen, 14 mars 1729 : LHAKo 53B 2647). C'est aussi le cas de Papius, qui avait longtemps été syndic du canton du Rhin-inférieur et qui fut au milieu des années 1750 présenté par Vienne, sur le contingent réservé au Cercle d'Empire de Bourgogne, pour un poste d'assesseur (lettre de Papius au Rhin-inférieur, présentée le 27 janv. 1756 : LHAKo 53B 1681). Autre signe très révélateur de l'affinité nouvelle qui lie la chevalerie d'Empire au TCI : la kyrielle de stagiaires issus de la chevalerie qui viennent au XVIIIe siècle passer un temps plus ou moins long à Wetzlar; voir Schmidt-Scharff (1934).

38. Voir Mauchenheim (1972), p. 432.

39. Sur la tendance à l'aristocratisation des assesseurs de la Chambre impériale, voir également Duchhardt (1977a), p. 117-118.

40. Certes, les premières réceptions d'assesseurs au sein de la chevalerie eurent lieu avant ce tournant (Eyben en 1688, Pürck en 1691) mais ce n'est qu'à partir de la deuxième décennie du XVIIIe siècle que le terme de « mode » est approprié.

41. Voir par exemple Mauchenheim (1972), p. 12-13 ou encore, dans le cadre d'un seul lignage, Mielke (1977), p. 117.

42. Le matériel statistique présenté sous forme de graphiques par Erwin Riedenauer ([1969], p. 132-151) propose une image très frappante de cette évolution.

43. Voir sa biographie dans Duchhardt (1977a), p. 112.

44. Lettre de Beaurieux au canton du Rhin-moyen, 15 nov. 1737 (StADa F2 41/10).

45. Des éléments biographiques dans Duchhardt (1977b), p. 107.

46. StADa F1 41/13.

47. 6 juin 1746 (LHAKo 53B 1939); formulation similaire dans la demande d'admission de Pütz, 25 févr. 1744 (copie dans LHAKo 53B 1713), de Schmitz le 28 mai 1744 (LHAKo 53B 1849) ou encore d'Ortmann le 27 juin 1756 (StADa F1 58/1).

48. Papius, par exemple, candidat à la réception en 1762 (lettre de Papius au canton de Steigerwald du 12 mars 1762, copies dans LHAKo 53B 1681 et StADa F1 58/5), était le gendre de l'assesseur Speckmann qui était déjà membre de la chevalerie d'Empire depuis 1749. Une vision plus générale de ces liens familiaux croissants entre les assesseurs du TCI : Praetorius (1937).

49. Les débats autour de la nomination d'Ingelheim ont vu également la chevalerie d'Empire employer toute son énergie à se faire à Vienne l'avocat du droit, par principe, des chevaliers à prétendre à cette fonction, la plus éminente du TCI, ou bien à faire défendre ce droit par des corporations qui lui était favorables (le chapitre cathédral de Mayence). Voir les correspondances à ce sujet dans LHAKo 53B 2647, ainsi que la note 37 ci-dessus. Sur la personnalité d'Ingelheim voir, outre mon article cité note 35, la notice dans la Neue Deutsche Biographie (vol. 10, 1974, p. 170-171).

50. Ainsi de la demande de Dünnwald, que le cercle de Souabe a même refusée en 1750 (lettre du 26 sept. 1750 au canton du Rhin-inférieur, LHAKo 53B 1138) - peut-être parce que les cercles franconien et rhénan lui avaient déjà fait parvenir un diplôme de réception daté du 2 janvier 
1749, donc antérieur à l'enregistrement de l'acceptation formelle de la part de la Souabe (voir LHAKo 53B 1939 et StADa F1 67/6). Mais en 1754 le cercle souabe a fini par donner son accord à la réception de Dünnwald (14 déc. 1754 : LHAKo 53B 2054 ou aussi 1269). Riedenauer (1969), p. 91 et 103 entre autres, souligne plus généralement les différends survenus entre la Souabe et les deux autres cercles de la chevalerie d'Empire.

51. En revanche je ne trouve aucune source qui confirme l'assertion, souvent présente dans la bibliographie (par exemple Riedenauer [1969], p. 94 et Stetten [1973], p. 86-87), selon laquelle les cantons auraient offert d'eux-mêmes aux juristes de les admettre en leur sein. Il ne fait certes pas de doute que des manœuvres d'approche aient pu être menées dans certains cas (voir par exemple la lettre de Geismar au directeur du canton du Rhin-supérieur Knebel von Katzenelnbogen, 22 févr. 1711, StADa F2 45/20 ; Jodoci à un correspondant inconnu, 14 mai 1723, StADa F1 49/9 ; Schmitz au canton du Rhin-inférieur, 28 mai 1744, LHAKo 53B 1849), mais de véritables accords préalables étaient naturellement exclus en raison de la nécessité de recueillir l'assentiment de tous les autres cercles.

52. Voir Cramer (1766), observatio MXLIX, p. 104-105.

53. Document du 25 févr. 1744 (LHAKo 53B 1713, aussi StADa F1 59/12, copie dans les deux cas) : Pütz prétend que depuis le début de sa carrière, d'abord comme conseiller au tribunal aulique de l'électeur palatin, puis du gouvernement et de la cour d'appel et finalement comme conseiller secret ainsi que dans ses missions auprès d'autres princes, il a eu à connaître - et il a pu faire avancer dans le bon sens - de nombreuses affaires concernant la chevalerie, et il a négocié à plusieurs reprises avec des conseillers et des directeurs de cantons chevaleresques.

54. Lettre de Fahnenberg au canton de Rhönwerra, 29 janv. 1793 (StADa F2 44/3). Sur sa biographie, voir Fahnenberg (1792), p. 200 sq.

55. Par exemple Geismar le 22 févr. 1711 (StADa F2 44/3), Ulmenstein le 20 oct. 1737 (StADa F2 41/10), Vogelius le 2 mai 1752 (LHAKo 53B 2054) ainsi qu'Ortmann le 27 juin 1756 (StADa F1 58/1) et Autenried le 4 juill. 1791 (StADa F2 44/6).

56. Autenried au canton d'Odenwald, 4 juill. 1791 (StADa F2 44/6).

57. Ainsi argumentèrent par exemple le canton de Rhönwerra au sujet de Speckmann (lettre de Rhönwerra au canton du Rhin-moyen, 6 juill. 1746, StADa F1 67/6), le canton du Rhin-moyen à propos de Cramer (2 nov. 1758, StADa F2 43/6), le canton de Rhönwerra en faveur de Fahnenberg (lettre de Rhönwerra au canton du Rhin-moyen, 9 avr. 1793, StADa F2 45/9).

58. On put craindre par exemple que la procédure concernant Franz à la fin des années 1720 prît du retard dès lors que le cercle souabe exigea que la demande fût traitée au cours d'une réunion générale des envoyés de tous les cantons (Generalkorrespondenztag). Les cantons rhénans répliquèrent par une argumentation habile: ils incitèrent à ne pas laisser l'affaire traîner si longtemps car le candidat aurait pu alors « en concevoir du dégoût » ce qui pouvait engendrer à Wetzlar de grands inconvénients pour la chevalerie d'Empire au lieu des bons services escomptés. Voir la correspondance dans StADa F2 45/9.

59. Lettre du canton de Gebirg à celui du Rhin-moyen, 8 oct. 1791 (StADa F1 37/15, copie).

60. Du canton d'Ortenau à celui de Donau, 6 févr. 1804 (StADa F1 37/15, copie).

61. La procédure était la suivante : le directoire général de la chevalerie informait les cantons qu'une demande d'admission lui était parvenue. Chaque canton interrogeait par écrit chacun de ses membres ou mettait l'affaire à l'ordre du jour d'une assemblée générale. Si la majorité des membres d'un canton était d'accord, puis si la majorité des cantons dans un cercle donnait un avis positif, le cercle en question envoyait au directoire général une réponse favorable. Ce n'est en règle générale que lorsque les trois cercles avaient donné leur feu vert que le diplôme de réception pouvait être rédigé. Voir Herrmann (1792), p. 73-83. Il est important de souligner que dans le cas des assesseurs du TCI on prononçait souvent une réception générale (sans inscription dans un canton particulier) ; voir Riedenauer (1969), p. 106.

62. Des éléments biographiques sur Geismar dans Duchhardt (1977a), p. 112 et 115 sq. 
63. Archives sur Geismar : StADa F2 45/20; sur Franz: StADa F2 45/9.

64. Lettre du canton du Rhin-moyen au canton du Rhin-inférieur, 11 janv. 1760 (LHAKo 53B 1044). D'autres argumentations similaires dans la lettre du Rhin-moyen au Rhin-inférieur du 22 déc. 1737 (LHAKo 53B 2029), dans celle du Rhin-inférieur à la Souabe, la Franconie, le Rhinsupérieur et le Rhin-moyen du 26 juin 1744 (LHAKo 53B 1849) et dans celle de Dünnwald au canton du Rhin-moyen du 21 août 1745 (StADa F1 41/13).

65. Les ouvrages et libelles du temps se sont fréquemment penchés sur la question de savoir si la réception d'assesseurs du TCI dans la chevalerie immédiate d'Empire visait à entraver leur impartialité, allant ainsi à l'encontre des règlements; voir par exemple Herrmann (1792), p. 28-29.

66. Voir Hellstern (1971), p. 34 et Riedenauer (1969), p. 102.

67. Seul le premier cas, celui de Geismar, a provoqué de fortes résistances contre le renoncement à la redevance d'admission : lettre du canton du Rhin-inférieur à celui du Rhin-supérieur, 24 nov. 1713 (StADa F2 45/20). Il finit pourtant par être incorporé gratuitement. Il semble que les assesseurs Vogelius, Papius et Ortmann, qui en 1752, 1762 et 1757 ont proposé d'eux-mêmes de s'acquitter des redevances habituelles, aient constitué des exceptions (LHAKo 53B 2054 et 1681, StADa F1 58/1).

68. Ainsi de Fahnenberg en 1793.

69. Deux exemples : lettre du canton du Rhin-inférieur à celui du Rhin-moyen du 15 janv. 1760 concernant Cramer (LHAKo 53B 1044); à propos de Dünnwald : lettre du canton du Rhin-inférieur à Dünnwald du 10 août 1755 (LHAKo 53B 1138).

70. Voir par exemple la lettre du canton de Donau à celui du Rhin-inférieur du 27 juin 1746 (LHAKo 53B 1849) ; la lettre de Donau au Rhin-inférieur du 14 déc. 1754 (ibidem, 1269) ; la lettre de Donau au Rhin-moyen du 21 mars 1759 (StADa F2 43/6). La liste établie à l'initiative de la Souabe et présentée le $1^{\text {er }}$ mai 1776 permet de constater que la réception gratuite des assesseurs du TCI s'était très largement imposée (StADa F2 40/7).

71. Les correspondances échangées en nov. 1758 se trouvent dans StADa F1 58/1. La procédure de réception de Reuß s'est déroulée d'une manière similaire; il fut également dispensé après coup de payer les sommes habituelles : lettre du canton du Rhin-inférieur à celui du Rhin-moyen, 15 janv. 1760 (LHAKo 53B 1044).

72. Ce fut par exemple le cas pour Fahnenberg dans les années 1790 : après que l'on eut longuement discuté de sa réception en tant qu'anobli, on décida finalement de s'abstenir de mentionner sa qualité de noblesse, neuve ou ancienne, dans le diplôme de réception ; voir StADa F2 44/3 et LHAKo 53B 1246, en particulier la lettre du canton de Donau exerçant la fonction de directoire général au canton du Rhin-supérieur le 4 déc. 1794 (StADa F2 44/3). L'assesseur Linden, présenté par la Bohême, constitue une exception : pour autant que les archives permettent de le savoir (StADa F1 52/6), il fut admis en 1800 en qualité de noble « nouveau ». Pour la biographie de Linden, voir Vahlkampf (1806), p. 494-495.

73. On peut certes constater que les impétrants du TCI ne marchandaient pas leurs promesses de faire l'acquisition d'un bien chevaleresque (par exemple Ulmenstein en 1737, StADa F2 41/10, Franz en 1728, StADa F2 45/9, Schmitz en 1744, LHAKo 53B 1849) mais en règle générale les chances pour que ces promesses soient effectivement tenues étaient assez minces. Pour autant, les sources n'offrent pas vraiment de fondement à la distinction entre membres "réels » et membres «à titre personnel ", pourtant souvent reprise par l'historiographie : on attendait des assesseurs comme des autres membres que leur appartenance à la chevalerie repose sur une base « réelle ", c'est-à-dire foncière. Le type du « personnaliste » qui en lieu et place d'un bien foncier met à la disposition du canton, sans intérêt, un capital de plusieurs milliers de florins et reçoit en retour le droit de siéger et de voter lors des assemblées cantonales (Herrmann [1792], p. 3 sq. ; Riedenauer [1969], p. 100-101) est un phénomène qui n'apparaît qu'à l'extrême fin du XVIIIe siècle. Voir Stetten (1973), p. 82-83. Il est révélateur que ce ne soit qu'en 1799 qu'un 
assesseur du TCI, Linden, ait - unique occurrence - demandé son admission comme membre purement personnel (StADa F1 52/6).

74. C'est par exemple le cas d'Eyben, qui acquit un domaine chevaleresque à Raunheim au milieu des années 1690 (StADa F1 42/16); Philipp Heinrich von Reuß possédait un bien chevaleresque à Steinheim, dans le canton d'Odenwald (lettre du canton de Rhönwerra à celui du Rhin-moyen, 30 janv. 1759, LHAKo 53B 1746) ; Linden en avait un à Wackenheim, dans le Rhin-supérieur (lettre de Linden au canton de Neckar-Forêt-Noire, non datée, StADa F1 52/6).

75. Depuis le statut commun de 1750 sur les réceptions, les preuves de noblesse avaient perdu en pratique toute signification. Le passage déterminant est le suivant: «En ce qui concerne les caractéristiques personnelles de l'impétrant, celui-ci doit être d'origine noble, ou bien avoir été élevé par sa Majesté impériale à la noblesse d'Empire, il est en outre tenu de prouver de manière crédible qu'aucun de ses ancêtres jusqu'au quatrième degré inclus n'a exercé une profession basse ni été de condition méprisable [...], il est toutefois permis au cercle chevaleresque par le biais duquel la réception est demandée, après en avoir fait communication aux autres cercles, de dispenser de ces conditions en fonction des mérites de l'impétrant et pour des raisons de la plus haute pertinence » (cité d'après Herrmann [1792], p. 17). Dans un recès de 1734, des clauses plus sévères concernaient encore la réception. En 1762, en dépit de l'objectif affiché de rendre les conditions d'admission plus difficiles, chaque cercle reçut définitivement la liberté d'accorder des dispenses en fonction des mérites du candidat (lettre du canton de Rhönwerra à celui du Rhin-moyen, 9 avr. 1793, StADa F2 44/3).

76. Voir par exemple les lettres du canton de Donau à celui du Rhin-moyen du 16 mars 1757 concernant Ortmann (StADa F1 58/1), du 20 déc. 1759 à propos de Reuß (LHAKo 53B 2746 et StADa F1 61/2), du 25 sept. 1793 au sujet de Fahnenberg (StADa F2 44/3). Seul ce dernier cas, celui de Fahnenberg en 1793-1794, a vu pour une fois le cercle rhénan s'employer lui aussi avec énergie à obtenir que l'arbre généalogique, conformément aux statuts, soit fourni par l'impétrant. Voir LHAKo 53B 1246, en particulier le compte-rendu du $1^{\text {er }}$ févr. 1794, ainsi que les lettres du canton du Rhin-supérieur à celui du Rhin-moyen le 14 févr. 1794 (StADa F2 44/3), du Rhin-moyen au Rhin-supérieur le 18 févr. 1794 (StADa F1 43/1) et du Rhin-supérieur au Rhin-moyen le 29 mars 1794 (StADa F2 44/3).

77. Voir par exemple la lettre de Dünnwald au canton du Rhin-moyen le 25 juin 1746 (StADa F1 41/13).

78. Avis favorables de la Franconie et de la Souabe (StADa F1 58/5) ainsi que du canton du Rhininférieur (LHAKo 53B 1681).

79. Lettres du canton du Rhin-moyen à ceux du Rhin-supérieur et du Rhin-inférieur, le 31 mars 1729 (StADa F2 45/9) et du Rhin-supérieur au Rhin-moyen le 27 mai 1729 (ibidem), comportant la ferme résolution de ramener enfin la Souabe vers de meilleures dispositions, car il n'était pas possible «que de telles expositions de notre opinion soient comprises par les personnes qualifiées siégeant dans les tribunaux suprêmes de l'Empire ».

80. On citera ici uniquement l'exemple de Kerner. Voir par exemple ses remarques dans la troisième partie de son Staatsrecht (Kerner [1789]), p. 99-100.

81. Voir par exemple Ludolf (1765).

82. Voir StADa F1 67/4. Naturellement, un impétrant de la stature de Spaur n'a pas fait savoir ses intentions d'emblée mais a utilisé un intermédiaire pour effectuer de premiers sondages, afin d'obtenir des assurances officieuses avant de franchir officiellement le pas. Le truchement fut en l'occurrence le baron Wilhelm von Edelsheim, membre de la chevalerie d'Empire (lettre d'Edelsheim au canton du Rhin-moyen présentée le 26 août 1763). Pour compléter la biographie de ce premier noble médiat à revêtir la dignité de Grand-juge, une précision intéressante, et jusqu'ici ignorée : son père, Johann Wilhelm Franz, avait demandé dès 1732 son admission au sein de la chevalerie immédiate auprès du cercle de Souabe ; le Generalkorrespondenztag de Nuremberg avait en 1734 donné une réponse positive (lettre du canton du Rhin-moyen aux conseillers 
chevaleresques, 29 août 1763) sans toutefois qu'il soit procédé à une réception ou à une prestation de serment. En se fondant sur cette décision antérieure, le canton du Rhin-moyen se vit en mesure de faire rédiger immédiatement le diplôme de réception sans effectuer les échanges habituels de correspondance avec les autres cercles, ce qui retarda l'acquisition, conforme aux statuts, d'un bien chevaleresque (lettre du canton du Rhin-moyen à Edelsheim, 29 août 1763 ; le diplôme est daté du 11 oct. 1763).

83. Pour sa biographie voir Diel (1957).

84. Ces indications sont fondées sur Ludolf (1765).

85. La proposition faite par le Wurtemberg est reproduite dans Herrmann (1792), p. 27-28.

86. Moser (1774), p. 418.

87. Voir Herrmann (1792), p. 28.

88. Ce conflit se noua autour de la personne du président Waldbott-Bassenheim, qui était en même temps directeur $\mathrm{du}$ canton $\mathrm{du}$ Rhin-moyen. Voir HHStAW Reichskanzlei Reichskammergerichtsvisitationsakten 352a, où l'on trouve également une série de mémoires issus de la chevalerie et rédigés dans les années 1764 et 1770-1771 sur la compatibilité des deux dignités. L'argumentation habile de la chevalerie est par exemple exposée dans une lettre à l'empereur du 14 févr. 1771 (ibidem). Voir également un mémoire de Bassenheim du $1^{\text {er }}$ août 1787 dans le Reichsritterschaftliches Magazin de Mader, livraison 10, p. 641.

89. Voir sur ce point les documents édités dans Moser (1775a), p. 11-24.

90. LHAKo 53B 186 : un volumineux Extractus Actorum sur les discussions de cette période.

91. C'est cette fois le Cercle d'Empire de Souabe qui avait pris l'initiative du débat (lettre du canton du Rhin-moyen à celui du Rhin-inférieur, 4 oct. 1720, LHAKo 53B 186). Les discussions à Ratisbonne et entre les cantons se poursuivirent jusqu'en 1723 (ibidem). Voir également Moser (1775a), p. 24.

92. Voir la lettre du canton de Gebirg à celui du Rhin-inférieur du 5 janv. 1723 (LHAKo 53B 186).

93. Un ouvrage d'un professeur de Tübingen paru juste avant cette reprise du débat et couplé à une sorte de description prévisionnelle du semestre d'hiver 1764-1765 (Tafinger [1764]) sousestime - à moins qu'il ne le néglige - un aspect fondamental des débats de ce temps : on escomptait que la chevalerie d'Empire offre une contribution financière au fonctionnement $\mathrm{du}$ TCI si elle voulait obtenir le droit d'y présenter des juges. Ce petit traité se contente donc d'observer que la chevalerie ne participe présentement d'aucune façon à la nomination des juges du TCI, « hac ipsa exclusione sua omnino contenta » (p. 11).

94. Document adressé par la chevalerie d'Empire à l'empereur, 28 oct. 1772 (HHStAW Mainzer Erzkanzlerarchiv, Reichskammergerichtsakten 382). Les avis des conseillers de l'empereur montrent que les représentations de la chevalerie ont recueilli un écho tout à fait favorable ; voir Kerner (1789), vol. 3, p. 95.

95. Voir Pfeiffer (1962), p. 270-279. Sur Tabor, voir en outre désormais Huschke (1974).

96. Dans la mouvance de ce désir d'égaler en tout point la chevalerie aux princes d'Empire, et à coup sûr sans volonté de s'opposer au TCI, émergèrent également des projets visant à mettre sur pied une instance d'appel commune à tous les sujets des chevaliers d'Empire - projets qui avaient toutefois fait l'objet au préalable, et à plusieurs reprises, d'un veto du TCI. Voir Vahlkampf (1806), p. 218-239.

97. Voir Press (1976b), p. 121, ainsi que les documents rassemblés dans Moser (1775b), p. 215-220.

98. Les raisons en sont très diversifiées. Elles comprenaient d'abord un simple calcul comptable : qu'est-ce qui était le plus avantageux pour la chevalerie ? Les traditionnels subsides caritatifs, ou bien les contributions découlant de l'inscription dans la Matricule d'Empire à quoi il fallait ajouter les coûts générés par l'envoi de délégués à la Diète d'Empire et aux assemblées des Cercles d'Empire? S'y adjoignaient des considérations politiques : être impliqué dans la décision de déclarer les guerres d'Empire aurait interdit aux cercles chevaleresques de Souabe et de Rhénanie, situés en première ligne, de sauvegarder leur neutralité - sans compter que 
l'inscription à la Matricule d'Empire aurait contraint à concéder aux états d'Empire un droit de regard sur l'état réel des biens relevant de la chevalerie d'Empire (voir Pfeiffer [1962], p. 186-187). La réticence à troquer une position singulière dans l'Empire pour la toute dernière place au sein du collège des princes à la Diète a pu également jouer un rôle (Pfeiffer [1962], p. 223). Des lignes de fracture durables se mirent en place dès la phase de négociation de la paix de Westphalie : le canton d'Odenwald devint l'avocat le plus ardent de l'accession au statut d'état d'Empire, une bonne partie du cercle souabe ainsi que les cantons d'Altmühl et Gebirg ses opposants les plus décidés. Press (1976b), p. 113, a raison de souligner que les efforts d'une partie de la chevalerie d'Empire pour accéder au statut d'état d'Empire «étaient un symptôme indubitable de la crise qui affectait la relation à l'empereur ».

99. On peut se faire une idée de cette crainte en consultant l'imposant ouvrage de Stephan Christoph Harpprecht (1727). Il n'est pas anodin que ce livre soit dédicacé à l'archevêqueélecteur de Mayence, Lothar Franz von Schönborn. De nombreux documents sur ce thème dans Moser (1775b).

100. Kerner (1789), vol. 3, p. 100.

101. LHAKo 53B 703.

102. Lettre du canton du Rhin-inférieur à celui du Rhin-moyen, 8 avr. 1804 (StADa F1 37/15). Branca n'était même pas encore en mesure de présenter ses quatre grands-parents et son arbre généalogique ; il fut pourtant admis en tant que noble d'ancienne extraction. Lettre du canton de Donau au Rhin-moyen du 29 déc. 1804 (ibidem).

103. De nombreux témoignages de ce discrédit de la chevalerie d'Empire dans (entre autres) Hofmann (1962), p. 175 et passim.

104. Voir Kirchner (1968) ainsi que Mager (1974).

105. Une opinion similaire dans Bader (1950), p. 171-172.

\section{INDEX}

Schlüsselwörter : Altes Reich, Reichsritterschaft, Reichskammergericht, Frühneuzeit, Gerichtsbarkeit und Gesellschaft

Mots-clés : Saint-Empire, chevalerie, tribunal de la Chambre Impériale, époque moderne, juridiction et société

\section{AUTEURS}

\section{HEINZ DUCHHARDT}

Heinz Duchhardt (Mayence) est président de la Fondation Max Weber. Pour plus d'informations, voir la notice suivante. 